\title{
An overview of burning mouth syndrome
}

Carmen Salerno $^{1}$, Dario Di Stasio ${ }^{1}$, Massimo Petruzzi ${ }^{2}$, Dorina Lauritano ${ }^{3}$, Enrica Gentile ${ }^{1}$, Agostino Guida ${ }^{1}$, Claudio Maio $^{1}$, Mariasofia Tammaro ${ }^{1}$, Rosario Serpico ${ }^{1}$, Alberta Lucchese ${ }^{1}$

${ }^{I}$ Multidisciplinary Department of Medical-Surgical and Odontostomatological Specialties, Second University of Naples, Via Luigi de Crecchio, 6, 80138 Naples, Italy, ${ }^{2}$ Section of Odontostomatology, University of Bari “Aldo Moro”, Bari, Italy,

${ }^{3}$ Department of Neuroscience and Biomedical Tecnologies. University of Milan- Bicocca Monza, Italy

\section{TABLE OF CONTENTS}

\author{
1. Abstract \\ 2. Introduction \\ 3. Pathophysiology hypothesis \\ 4. Diagnosis \\ 5. Treatment \\ 5.1. Topical treatment \\ 5.2. Sistemic treatment \\ 6. Summary and perspectives \\ 7. Acknowledgements \\ 8. References
}

\section{ABSTRACT}

Burning mouth syndrome (BMS) is characterised by the presence of a burning sensation in the oral mucosa in the absence of any clinically apparent mucosal sign. It occurs more commonly in older women and often affects the tongue tip and lateral borders, lips, and hard and soft palates. Besides the burning sensation, patients with BMS may complain of unremitting oral mucosal pain, dysgeusia, and xerostomia. The exact pathophysiology of primary BMS remains unknown. A major challenge for the clinician is the treatment of BMS: identifying possible causative factors is the first step, but BMS is often idiopathic. Drug therapy, in addition to behavioural therapy and psychotherapy, may help to eliminate the symptoms. Considering the growing incidence of BMS in older people, further research is required to determine the true efficacy of current management strategies for patients with this disorder.

\section{INTRODUCTION}

Burning mouth syndrome (BMS) is a chronic pain disorder characterised by burning, stinging, and/or itching of the oral cavity in the absence of any apparent organic disease. Often, symptoms involve the tongue, with or without extension to the lips and oral mucosa $(1,2)$. BMS can be accompanied by dysgeusia and subjective xerostomia. Its onset is spontaneous and the syndrome has a clear predisposition in geriatric patients, mostly women $(3,4)$. Its secondary form has been associated with various conditions, including thyroid disease, psychiatric illnesses, oral infections, drug use, dental treatment, and vitamin/mineral deficiencies $(5,6)$. It was first categorised as a distinct disease in 2004 by the International Headache Society, which defined primary BMS as "an intraoral burning sensation for which no medical or dental cause can be found." Current diagnostic criteria consist of daily persistent pain in the mouth with a normal oral mucosa, after exclusion of local and systemic diseases (7). The epidemiological data on BMS are poor, with reported prevalence rates in the general population varying from 0.7 to $15 \%(8,9)$.

\section{PATHOPHYSIOLOGY HYPOTHESIS}

"Primary" or essential or idiopathic BMS, for which no organic local or systemic cause can be identified, likely has a neuropathological cause. "Secondary BMS" is a variant resulting from local or systemic pathological conditions. Various conditions may lead to secondary BMS (e.g. lichen planus, candidiasis, coeliac disease) (10-12), hormonal disturbances, psychosocial stressors, vitamin or nutritional deficiencies, diabetes, dry mouth, contact allergies, galvanism, parafunctional habits, and cranial nerve injuries. Evidence suggests a neuropathic mechanism of the disease (13). Oral mucosal biopsies of patients with BMS demonstrated a decreased density of epithelial nerve fibres, as well as axonal derangement, indicating a potential role for peripheral small-fibre sensory neuropathy (14). Blink reflex abnormalities, suggesting a brain stem pathology or peripheral trigeminal neuropathy, as well as sensorial modifications, such as hypoesthesia, probably due to peripheral small-fibre neuropathy, have also been described in patients with BMS (15). A study by Lin et al. (16) showed that BMS patients had significantly higher frequencies of $\mathrm{Hb}$, iron, and vitamin B12 deficiencies, abnormally elevated blood homocysteine levels, and serum gastric parietal cell antibody positivity, compared with healthy control individuals. A study by Corsalini et al. showed a high percentage of temporomandibular disorders (TMD) in BMS patients that could be caused by an overload of the masticatory system: anxiety and restlessness were frequently referred to in the anamnestic interview and wear facets were often observed 
during the clinical evaluation of these patients, as an effect of the stress associated with the primary disease (17). It is probable that the stress condition could explain the onset of parafunctional habits (the main causes of TMD) in these patients. However, it is also possible that the same neuropathic alterations assumed for BMS could be involved in the parafunctional habits too. Literature reports describe cases of drug-induced BMS (e.g. topiramate, carbidopa/levodopa) $(18,19)$. Although these cases may not truly represent primary BMS, it may be important to understand drug-induced cases and other causative factors for oral burning sensations. Sleep dysfunction may also play a role in BMS (20). In a cross-sectional, case-controlled study, patients with BMS reported a higher degree of sleep disturbance than age- and gender-matched controls with various oral conditions. It has been suggested that sleep dysfunction may be a risk factor and possible target for the treatment of BMS (21). Psychological and psychiatric disorders can be found in up to $85 \%$ of patients, with anxiety, depression, hypochondria, cancerophobia, and insomnia being the most common diagnoses $(22,23)$. In a retrospective review, patients affected by BMS also presented with hypertension (33\%), gastro-oesophageal reflux disease $(29 \%)$, autoimmune disorders $(22 \%)$, hypercholesterolaemia (22\%), thyroid disorders (14\%), and anaemia (2\%) (24). Evidence for chorda tympani hypofunction in BMS has been presented recently (25). Of 22 BMS patients examined with an electrical quantitative sensory testing apparatus, $82 \%$ demonstrated chorda tympani dysfunction (59\% unilateral and $23 \%$ bilateral). This supports the hypothesis that tonic inhibition of the sensory pathways by gustatory activity is dysfunctional in BMS patients (26). In addition to changes at the peripheral level, there are data illustrating the involvement of the central nervous system, and interactions with the peripheral nervous system (27). Grushkaet et al. (28) proposed that BMS could be correlated with hyperactivity of both the sensory and motor components of the trigeminal nerve, due to a loss of central inhibition, as a result of taste damage in the chorda tympani and/or the glossopharyngeal nerves. That may result in increased activity in the muscles of mastication and the intrinsic muscles of the tongue. Studies into the dopaminergic pathway of central pain modulation have also been conducted. Hagelberg (29) demonstrated a reduction in endogenous dopamine levels in the putamen in burning mouth patients, consistent with a theory of presynaptic dysfunction of the nigrostriatal dopaminergic pathway of central pain modulation in BMS. A recent study indicated that thermal stimulation in BMS patients was associated with increased cerebral blood flow, as observed on functional magnetic resonance imaging (30). Moreover, the finding that oestrogens work as neuroprotectants of the nigrostriatal dopaminergic system $(31,32)$ could explain the correlation between menopause and this disorder. The different patterns of MUC1 and TLR-2 expression between BMS and oral lichen planus (OLP) suggest that the pathogenesis of BMS is not inflammation-related. A study by Hong-Seop Kho et al. analysed the expression levels of MUC1 and TLR-2 in BMS patients, and compared them with those of controls and OLP patients. The relationships between the expression levels of MUC1 and TLR-2 and the levels of salivary pro-inflammatory cytokines were also investigated. They reported that increased expression of MUC1 may play a role in the development and/or progression of BMS (33).

\section{DIAGNOSIS}

For the diagnosis of BMS, a careful analysis of all symptoms in each patient is required. The characteristic symptoms of burning mouth or pain should be felt deep within the oral mucosa, continuously for at least 4-6 months, almost every day; they are never worsened, but almost always are relieved by eating or drinking. Other symptoms such as oral dysgeusia, a spontaneous metallic taste, xerostomia, and the presence of abnormal sensory/chemo-sensory feelings, mood changes, interruptions, and specific personality traits of patients may also help identify BMS. Additionally, patients with BMS show no clinical sign or objective basis of any pathology of the oral mucosa (34). Thus, the clinical diagnosis is based on a thorough examination, the patient's medical history, and a detailed analysis of the data obtained from physical and laboratory examinations. A clinical classification of BMS has been proposed based on a symptoms framework $(27,35)$. Three types of BMS have been recognised: Type 1 is characterized by a pain-free wake up, with burning sensation gradually increasing in severity during the day, and reaching its peak intensity by the evening. This type affects approximately $35 \%$ of patients and is linked to systemic disorders, such as nutritional deficiencies and diabetes mellitus. In Type 2, symptoms are continuous throughout the day. Patients fall asleep with difficulty. This type occurs in about $55 \%$ of patients and is usually associated with psychological disorders. Type 3 BMS is characterised by intermittent symptoms, with pain-free periods during the day. Generally, these patients constitute $10 \%$ of total patients and show allergic reactions $(27,35)$.

\section{TREATMENT}

In the management of patients affected by BMS, it is important to exclude other possible causes, such as local or systemic causes (galvanic current, mechanical irritation, parafunctions, allergic reactions, anaemia, mineral or nutritional deficiencies, infections, drugs and metabolic, genitourinary, gastrointestinal, neurological, and psychiatric disorders). It is also advisable to change, in consultation with a specialist, drug therapies that may interfere with the onset of BMS, such as angiotensin-converting-enzyme inhibitors. Curing BMS, however, remains difficult despite the different classes of drugs that have been used. The variable rate of response to medical therapy is most likely related to the multifactorial nature of idiopathic BMS. The aim of treatment is mainly management of the disease, as a chronic neuropathy (7). Patients with BMS show a response to treatment with anxiolytics, anticonvulsants, antidepressants, atypical antipsychotics, histamine receptor antagonists, and dopamine agonists. Other treatments that have shown efficacy include clonazepam, herbal supplementation with Catuama, aloe vera, alpha-lipoic acid in combination with gabapentin, capsaicin wash, acupuncture, and alpha-lipoic acid alone (20).

\subsection{Topical treatment}


Capsaicin, clonazepam, lidocaine, benzydamine hydrochlorate at $0.15 \%$, and aloe vera have been used topically for the treatment of BMS. Capsaicin acts on the sensory afferent neurons and topical capsaicin can be used as a desensitising agent or an analgesic for the treatment of oral mucosal burning (34). However, capsaicin is difficult for many patients to accept because of its taste. Moreover, it generally causes an increase in the burning sensation at the beginning of treatment (36). In a prospective study with 30 patients with BMS, a capsaicin rinse $(0.02 \%)$ significantly reduced VAS from baseline over placebo (20). However, there were seven drop-outs, which may suggest limitations to the treatment due to side effects (37). Clonazepam is an agonist of gamma-aminobutyric acid. Local application of clonazepam may lessen the sting but its use has systemic adverse effects (34). Lozenges of clonazepam (oral dissolution of a $1 \mathrm{mg}$ tablet for $3 \mathrm{~min}$ and then expectorate three times a day) are effective in patients with predominantly peripheral BMS (38). The efficacy of diazepam and clonazepam for the treatment of BMS was compared in a retrospective review. Barker et al. reported that a higher percentage of patients responded to clonazepam (71.4.\%), but patients responded also to diazepam (55.1.\%) and there was no significant difference between the two treatments (39). Lidocaine and $0.15 \%$ benzydamine hydrochlorate have anaesthetic effects and are anti-inflammatories. These two agents are used as a mouthwash to reduce pain or burning symptoms. However, they cannot be used as an effective therapy because of the short duration of their analgesic effect (40). In a study by Lopez-Jornet et al, $0.5 \mathrm{~mL}$ aloe vera gel, at $70 \%$ applied on the sore areas of the tongue three times a day in combination with a tongue protector, reduced burning and pain (41).

\subsection{Systemic treatment}

It has been observed that the use of systemic capsaicin $(0.25 \%$, three times per day, for 30 days) leads to a significant improvement in symptoms of pain compared with a placebo group (34). However, it cannot be used in long-term therapy because after 4 weeks of treatment, $32 \%$ of patients reported gastric pain (42). Systemic clonazepam ( $0.5 \mathrm{mg} /$ day) has proven effective in reducing the symptoms of BMS. Amos et al. (43) used combination therapy with topical and systemic clonazepam. Patients were asked to dissolve the clonazepam tablet $(0.5 \mathrm{mg} /$ tablet, three times daily) orally before swallowing and were followed for a period of 6 months. Of the patients, $80 \%$ obtained more than a $50 \%$ reduction in pain during the treatment period. One-third of the patients had complete resolution of pain, suggesting the effectiveness of the combined treatment.

Alpha-lipoic acid is a powerful antioxidant that has mitochondrial coenzyme neuroprotective effects. It is also an antioxidant that can increase the levels of intracellular glutathione and eliminate free radicals. Several reports have described alpha-lipoic acid treatment at $600 \mathrm{mg}$ daily for more than 2 months in the treatment of patients with BMS (44). Other studies concluded that alpha-lipoic acid was not efficacious in BMS treatment.

Maina (45) evaluated the efficacy and tolerability of amisulpride (50 mg/day) and selective serotonin reuptake inhibitors - paroxetine $(20 \mathrm{mg} /$ day $)$ and sertraline $(50 \mathrm{mg} /$ day $)$ - in the treatment of BMS for 8 weeks. They found that all three treatments were effective in reducing pain and burning. ${ }^{8}$ Amisulpride gave the best results in the short term, and better compliance than the other two drugs. There were no significant adverse effects. The results of this study seem to suggest that they were equally effective and equally well-tolerated in the short-term treatment of BMS. It was also seen that, in combination with drug therapy, weekly 1 -h sessions of cognitive behavioural therapy lasting 12-15 weeks reduced the symptoms of BMS significantly in all patients in the study group compared with the placebo control (46). However, some studies have documented high placebo response rates (16). These results provide the basis for the design of future studies to evaluate therapies for BMS. To obtain strong evidence of efficacy in treatment of BMS, adequately sized prospective multicentre studies are essential. These studies need to obtain better results for the use of a substance than the placebo, including a standard protocol of instructions, and a long period of follow-up (13).

\section{SUMMARY AND PERSPECTIVES}

BMS is a relatively common chronic intraoral pain disorder in peri-/postmenopausal women, classically characterised by intractable burning that may be associated with dysgeusia and xerostomia. Its pathogenesis is related to a complex interplay of central and/or peripheral neural pain pathways. The aetiology of BMS is multifactorial and the secondary form of BMS should be diligently sought and treated (5). The aetiopathogenesis of BMS is complex: local, systemic, and/or psychological factors are involved in generating the painful oral burning symptoms. BMS has also been found to be associated with peripheral nerve damage and dopaminergic system disorders. A major challenge for the clinician is the treatment of BMS: identifying the possible causative factors is the first step, because treatment or elimination of these factors can lead to significant clinical improvement in the painful oral burning symptoms. However, this condition is often idiopathic; in this case, drug therapy should be instituted. Additionally, psychotherapy and behavioural feedback may help to eliminate BMS symptoms (6). In conclusion, further research is required to determine the true efficacy of current management strategies for patients with this disorder.

\section{ACKNOWLEDGEMENTS}

All the authors contributed equally to this article.

\section{REFERENCES}

1. F.O. Netto, I.M. Diniz, S.M. Grossmann, M.H. de Abreu, M.A. do Carmo, M.C. Aguiar: Risk factors in burning mouth syndrome: a case-control study based on patient records. Clin Oral Investig 15, 571-575 (2011)

2. P.J. Lamey, A.B. Lamb: Prospective study of aetiological factors in burning mouth syndrome. Br Med J (Clin Res Ed) 296, 
$1243-1246(1988)$

3. J.H. Meurman, L. Tarkkila, A. Tiitinen: The menopause and oral health. Maturitas 63, 56-62 (2009)

4. JE. Fantasia: Diagnosis and treatment of common oral lesions found in the elderly. Dent Clin North Am 41, 877-890 (1997)

5. Z. Yilmaz, T. Renton, Y. Yiangou, J. Zakrzewska, I.P. Chessell, C. Bountra, P. Anand: Burning mouth syndrome as a trigeminal small fibre neuropathy: Increased heat and capsaicin receptor TRPV1 in nerve fibres correlates with pain score. $J C l i n$ Neurosci 14, 864-871 (2007)

6. M. Grushka, J.B. Epstein, M. Gorsky: Burning mouth syndrome and other oral sensorydisorders: a unifying hypothesis. Pain Res Manag 8, 133-135 (2003)

7. G.E. Gurvits, A. Tan: Burning mouth syndrome. World J Gastroenterol 19, 665-672 (2013)

8. J.A. Lipton, J.A. Ship, D. Larach-Robinson: Estimated prevalence and distribution of reported orofacial pain in the United States. J Am Dent Assoc 124, 115-121 (1993)

9. T. Tammiala-Salonen, T. Hiidenkari, T. Parvinen: Burning mouth in a Finnish adult population. Community Dent Oral Epidemiol 21, 67-71 (1993)

10. M. Srinivasan, K.N. Kodumudi, S.L. Zunt: Soluble CD14 and toll-like receptor-2 are potential salivary biomarkers for oral lichen planus and burning mouth syndrome. Clin Immunol 126, 31-37 (2008)

11. H. Terai, M. Shimahara: Glossodynia from Candida-associated lesions, burning mouth syndrome, or mixed causes. Pain Med $11,856-860(2010)$

12. A. Lucchese, A. Guida, R. Serpico: Glossodynia and coeliac disease. Immuno pharmacol Immunotoxicol 34, 247-249 (2012)

13. M. Uten-Shorrer, J.M. Kelley, S.T. Sonis, N,S. Treister: Placebo effect in burning mouth syndrome: a systematic review. Oral Dis 20, 1-6 (2014)

14. G. Lauria, A. Majorana, M. Borgna, R. Lombardi, P. Penza, A. Padovani, P. Sapelli: Trigeminal small fiber sensory neuropathy causes burning mouth syndrome. Pain 115, 332-337 (2005)

15. H. Forssell, S. Jääskeläinen, O. Tenovuo, S. Hinkka: Sensory dysfunction in burning mouth syndrome. Pain 99, 41-47 (2002)

16. H.P. Lin, Y.P. Wang, H.M. Chen, Y.S. Kuo, M.J. Lang, A. Sun: Significant association ofhematinic deficiencies and high blood homocysteine levels with burning mouth syndrome. J Formos Med Assoc 112, 319-325 (2013)

17. M. Corsalini, D. Di Venere, F. Pettini, D. Lauritano, M. Petruzzi: Temporomandibular disorders in burning mouth syndrome patients: an observational study. Int J Med Sci 10, 1784-1789 (2013)

18. D.I. Friedman: Topirimate-induced burning mouth syndrome. Headache 50, 1383-1385 (2010)

19. E.A. Coon, R.S. Laughlin: Burning mouth syndrome in Parkinson's disease: dopamine ascure or cause? J Headache Pain 13, 255-257 (2012)

20. L. 4th. Charleston: Burning mouth syndrome: a review of recent literature. Curr Pain Headache Rep 17, 336 (2013)

21. N. Chainani-Wu, E. Madden, S. Jr. Silverman: A case-control study of burning mouthsyndrome and sleep dysfunction. Oral Surg Oral Med Oral Pathol Oral Radiol Endod 112, 203-208 (2011)

22. O. Komiyama, H. Nishimura, Y. Makiyama, T. Iida, R. Obara, M. Shinoda, M. Kobayashi, N. Noma, O. Abe, A. De Laat, M. Kawara: Group cognitive behavioral intervention for patients with burning mouth syndrome. J Oral Sci 55, 17-22 (2013)

23. F.T. De Souza, A.L. Teixeira, T.M. Amaral, T.P. dos Santos, M.H. Abreu, T.A. Silva, A. Kummer: Psychiatric disorders in burning mouth syndrome. J Psychosom Res 72, 142- 146 (2012)

24. G.D. Klasser, J.B. Epstein, D. Villines: Diagnostic dilemma: the enigma of an oral burning sensation. J Can Dent Assoc 77, $146(2011)$

25. E. Liav, B. Kamran, R. Schaham, R. Czerninski, R.H. Gracely, R. Benoliel: Evidenceof chorda tympani dysfunction in patients with burning mouth syndrome. J Am Dent Assoc 138, 628-633 (2007)

26. L.L. Patton, M.A. Siegel, R. Benoliel, A. De Laat: Management of burning mouth syndrome: systematic review and management recommendations. Oral Surg Oral Med Oral Pathol Oral Radiol Endod 103, 1-13 (2007)

27. A. Scala, L. Checchi, M. Montevecchi, I. Marini, M.A. Giamberardino: Update on burning mouth syndrome: overview and patient management. Crit Rev Oral Biol Med 14, 275-291 (2003)

28. M. Grushka, J.B. Epstein, M. Gorsky: Burning mouth syndrome and other oral sensory disorders: a unifying hypothesis. Pain Res Manag 8, 133-135 (2003)

29. N. Hagelberg, H. Forssell, J.O. Rinne, H. Scheinin, T. Taiminen, S. Aalto, S. Luutonen, K. Någren, S. Jääskeläinen: Striatal dopamine D1 and D2 receptors in burning mouth syndrome. Pain 101, 149-154 (2003)

30. R.J. Albuquerque, R. de Leeuw, C.R. Carlson, J.P. Okeson, C.S. Miller, A.H. Andersen: Cerebral activation during thermal stimulation of patients who have burning mouth disorder: an fMRI study. Pain 122, 223-234 (2006)

31. T.M. Gajjar, L.I. Anderson, D.E. Dluzen: Acute effects of estrogen uponmethamphetamine induced neurotoxicity of the nigrostriatal dopaminergic system. J Neural Transm 110, 1215-1224 (2003)

32. D.B. Miller, S.F. Ali, J.P. O'Callaghan, S.C. Laws: The impact of gender and estrogen on striatal dopaminergic neurotoxicity. Ann N Y Acad Sci 844, 153-165 (1998)

33. H.S. Kho, J.Y. Chang, Y.Y. Kim, Y. Kim: MUC1 and Toll-like receptor-2 expression in burning mouth syndrome and oral lichen planus. Arch Oral Biol 58, 837-842 (2013)

34. A. Sun, K.M. Wu, Y.P. Wang, H.P. Lin, H.M. Chen, C.P. Chiang: Burning mouth syndrome: a review and update. J Oral Pathol Med 42, 649-655 (2013)

35. P. López-Jornet, F. Camacho-Alonso, P. Andujar-Mateos, M. Sánchez-Siles, F. Gómez-Garcia: Burning mouth syndrome: an update. Med Oral Patol Oral Cir Bucal 15, 562-568 (2010)

36. J.B. Epstein, J.H. Marcoe: Topical application of capsaicin for treatment of oral neuropathic pain and trigeminal neuralgia. 
Oral Surg Oral Med Oral Pathol 77, 135-140 (1994)

37. F.J. Silvestre, J. Silvestre-Rangil, C. Tamarit-Santafé, D. Bautista: Application of a capsaicin rinse in the treatment of burning mouth syndrome. Med Oral Patol Oral Cir Bucal 17, 1-4 (2012)

38. C. Grémeau-Richard, C. Dubray, B. Aublet-Cuvelier, S. Ughetto, A. Woda: Effect of lingual nerve block on burning mouth syndrome (stomatodynia): a randomized crossover trial. Pain 149, 27-32 (2010)

39. K.E. Barker, M.D. Batstone, N.W. Savage: Comparison of treatment modalities in burning mouth syndrome. Aust Dent J 54, 300-305 (2009)

40. A. Sardella, D. Uglietti, F. Demarosi, G. Lodi, C. Bez, A. Carrassi: Benzydamine hydrochloride oral rinses in management of burning mouth syndrome. A clinical trial. Oral Surg Oral Med Oral Pathol Oral Radiol Endod 88, 683-686 (1999)

41. P. López-Jornet, F. Camacho-Alonso, D. Molino-Pagan: Prospective, randomized, double-blind, clinical evaluation of Aloe vera Barbadensis, applied incombination with a tongue protector to treat burning mouth syndrome. J Oral Pathol Med 42, 295301 (2013)

42. M. Petruzzi, D. Lauritano, M. De Benedittis, M. Baldoni, R. Serpico: Systemic capsaicin for burning mouth syndrome: shortterm results of a pilot study. J Oral Pathol Med 33, 111-114 (2004)

43. K. Amos, S.C. Yeoh, C.S. Farah: Combined topical and systemic clonazepam therapyf or the management of burning mouth syndrome: a retrospective pilot study. J Orofac Pain 25, 125-130 (2011)

44. A. Sardella, G. Lodi, F. Demarosi, C. Bez, S. Cassano, A. Carrassi: Burning mouth syndrome: a retrospective study investigating spontaneous remission and response to treatments. Oral Dis 12, 152-155 (2006)

45. D.R. Cavalcanti, F.R. da Silveira: Alpha lipoic acid in burning mouth syndrome: a randomized double-blind placebocontrolled trial. J Oral Pathol Med 38, 254-261 (2009)

46. J. Bergdahl, G. Anneroth, H. Perris: Cognitive therapy in the treatment of patients with resistant burning mouth syndrome: a controlled study. J Oral Pathol Med 24, 213-215 (1995)

Abbreviations: BMS: burning mouth syndrome; TMD: temporomandibular disorders; OLP: oral lichen planus

Key Words: Burning Mouth Syndrome, Chronic Pain, Oral Pain, Oral Health, Review

Send correspondence to: Dario Di Stasio, Multidisciplinary Department of Medical-Surgical and Odontostomatological Specialties; via L. De Crecchio, 680138 Naples, Italy, Tel: 00390815667674, Fax: 00390815667674, E-mail: dario.distasio@unina2.it

Running title: An overview of burning mouth syndrome 